\title{
Enlightening a Co-Located Community with a Semi-Public Notification System
}

\author{
Goldie B. Terrell \\ Department of Computer Science \\ and Center for $\mathrm{HCl}$, Virginia Tech \\ Blacksburg VA 24061-0106 \\ +1540 231-7409 \\ gterrell@vt.edu
}

\author{
D. Scott McCrickard \\ Department of Computer Science \\ and Center for $\mathrm{HCl}$, Virginia Tech \\ Blacksburg VA 24061-0106 \\ +1540 231-6698 \\ mccricks@cs.vt.edu
}

\begin{abstract}
This work seeks to strengthen interaction within a research community through a centrally-located physical device that presents online presence information in a semi-public space. The device uses a map metaphor to represent a set of connected labs. As people move within the lab, those who wish to interact with lab users can use the display to guide their interaction approaches, by supporting educated guesses as to arrivals, departures, and work patterns. The paper reports on the lessons learned about the device's characteristics, and provides anecdotes and observations on ways in which this type of device can improve communication and enhance community.
\end{abstract}

\section{Categories and Subject Descriptors}

H5.2 [Information interfaces and presentation]: User Interfaces. Graphical user interfaces

\section{General Terms}

Design, Experimentation, Human Factors.

\section{Keywords}

Awareness, notification systems, ubiquitous displays

\section{INTRODUCTION}

Instant messaging has shifted from merely a fun tool for planning social events to also an essential work tool for coordinating deadlines and planning meetings. In response, the research community has been investigating IM and similar desktop tools helpful to the business world. Our work moves off the desktop and beyond the domain of single-user tools, investigating how tools integrated into a collaborative environment can help not only individuals (as exemplified in [1]) but also communities of researchers in shared spaces to connect and communicate.

The desire to create an off-the-desktop system is inspired by the "space vs. place" dynamic introduced by Harrison and Dourish

Permission to make digital or hard copies of all or part of this work for personal or classroom use is granted without fee provided that copies are not made or distributed for profit or commercial advantage and that copies bear this notice and the full citation on the first page. To copy otherwise, or republish, to post on servers or to redistribute to lists, requires prior specific permission and/or a fee.

CSCW '06, November 4-8, 2006, Banff, Alberta, Canada.

Copyright 2006 ACM 1-59593-249-6/06/0011 ...\$5.00. with respect to objects and technologies located in media spaces, MUDs, and similar physical and virtual places [8]. They sought to explore how a culturally rich place, not a physical space, is often more valuable to capture in design. Others have built upon this idea, e.g., using multiple monitors to transform space into place [4]; and supporting remote collaborators in a space to help enhance place [9]. We seek to extend the "space vs. place" dynamic to understand how an interface can help enrich our collaborative environment - not only through large group interactions centered around a device but, more importantly, through individual or small group interactions with freelyavailable (without requiring badges or locators), automaticallycollected, constantly-accessible information about collaborators.

We ground our interface within the domain of semi-public displays, interfaces intended to primarily benefit a small, familiar group. Huang and Mynatt introduced the concept to explore avenues for how information could be presented on a large display as a focal point for activities [6]. They hypothesized that these displays mitigate relevance and privacy concerns compared to designs for large, loosely connected groups. Follow-up work specifically looked at IM information on large, shared displays [7]. We targeted an off-the-desktop system for improving community through a focused but secondary information source and a small, non-monitor-based presentation style, balancing desires for an attractive off-the-desktop system with the need for a functional tool for readily communicating presence information.

Starting from this base, the goal of this project was to create a notification system that would alert users in our lab of the online availability of their lab colleagues. We were inspired by the work of McPhail, who used phidgets [3] to develop Buddy Bugs, an off-the-desktop IM display tool. Her project uses a "bugs-on-aleaf" metaphor, using ceramic bugs to represent online buddies [11]. This tool was presented as an off-the-desktop extension to typical IM tools, and we sought to take the idea to the next level by providing IM status and history for a community of workers. Since many members are dependent on others for their work, opportunistic guidance on the next task to undertake - start a meeting, interrupt a colleague, sit down to work independentlycan be made during transitions. Enhancing interaction opportunities should increase lab efficiency and output.

Our root concept required a centrally-located display of the current online status of users in the lab, seeking to ensure easy reaction to this information and minimal diversion from users' primary tasks. Our solution, Online Enlightenment (OE), provides 
a semi-public ubiquitous system for monitoring online presence of lab members on MSN Messenger (Fig. 1). In its current form, deployed in our lab for over four months, OE has caricatures of each person in the lab on buttons in a layout that mirrors the lab, with accompanying 3-way LEDs showing online presenceproviding at-a-glance online presence information about lab users. An LCD screen at the top of the device displays a lab user's name, status, and time since last MSN Messenger status change. When a lab user's button is pushed or status changes, the display shows the user's updated data. This gives information not available in the standard Messenger interface. In this paper, we give an overview of the design and implementation of $\mathrm{OE}$ (see also [5]) and report on usage of $\mathrm{OE}$ and speculate on reasons for reactions.

\section{DESIGN AND IMPLEMENTATION}

Many of the design goals came from a participatory design session, held with regular and sporadic users of the lab. However, our design could not possibly incorporate all of the ideas presented at the session. We selected the core desires for the lab users and lab visitors, with an eye to practicality.

\subsection{Design Goals}

In considering our design, we first thought about how effectively existing instant messaging (IM) tools meet the needs of the lab community. Almost all of the lab users already use IM to communicate with friends and are generally happy with it for their individual needs, but there is little support within most IM tools for establishing communities of users. We speculate that a close-knit community of users in a semi-public place would benefit from knowing about their colleagues - thus transforming a space where users work into a place that fosters interaction and collaboration (in keeping with the sentiment introduced in [8]). In addition, some IM tools store no history; knowing when status changed may help to determine current activities.

By their very nature, desktop IM tools create an insular working environment, where collaborations are one-on-one (though often multiple one-on-one conversations may be occurring simultaneously, and others may be interacting with an IM conversant). Acquiring and maintaining knowledge of presence for users through pop-ups and blinking windows has questionable value for users at the desktop, but is even more challenging as users move about a lab environment physically interacting with each other. Even the off-the-desktop tools developed to work with IM clients, such as those developed and referenced in [1], generally are intended to be used by a single person, not a group.

Our design sought to build community through an off-the-desktop system that would simplify use and lessen distraction while raising the amount of information available to a moderately-sized research team housed in a shared lab. We felt this would meet our community-related goals: allowing those that come to the lab to acquire and retain knowledge about lab users while opportunistically interacting with them in appropriate ways with minimal interruption to all concerned. The goal of the design was to build a device that provided easy access to information regarding the availability of lab users while providing insight on the lab to visitors. As such our primary stakeholders were lab users who needed to be able to find out when their colleagues would be available for collaboration, with lab visitors as secondary stakeholders who would like to find (and learn about) lab users and community.

While there has been success in creating image-based displays on publicly accessible spaces (e.g., $[2,6,7,12]$ ), generally they are meant to be a focal point of activities and conversations. In contrast, we seek to enable interactions through other meansface-to-face, IMing, phone calls, etc. In a quick glance, a user or group of users should be able to absorb all available information and make an informed decision on a course of action. However, our desire to use a non-digital low-bandwidth display implies that care must be taken to select appropriate information for the display. And as with many new technologies, integration into the work space must be done with care to ensure acceptance.

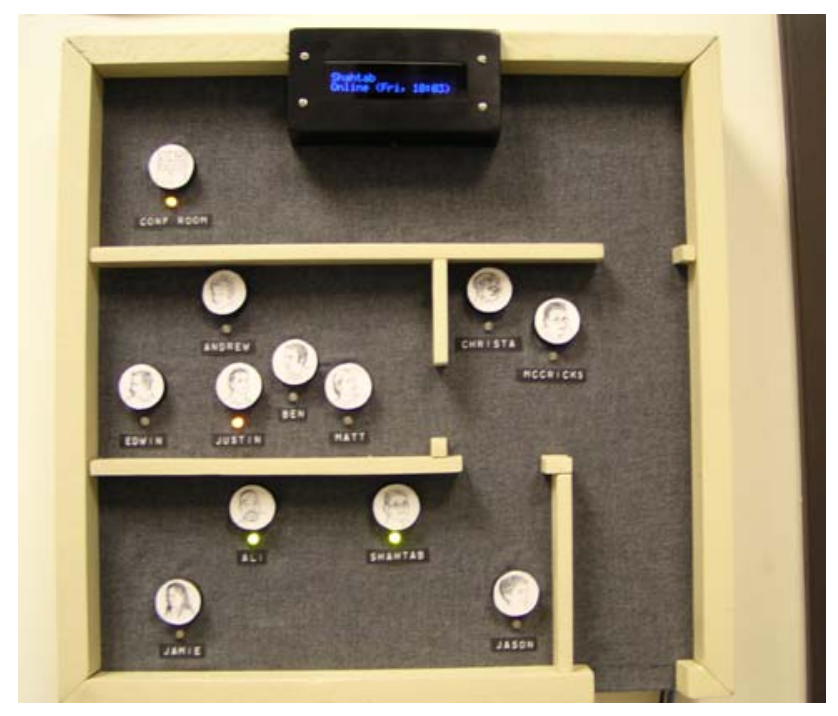

Fig. 1. Online Enlightenment. The appearance reflects the layout of the lab. Caricatures spaced on position provide three ways to identify users: image, name, and location.

\subsection{Feature Selection}

The map metaphor helps both regular users and occasional visitors establish and reinforce relationships between members of the lab. It has been used in other applications to guide users to the location and grouping of colleagues (e.g., [10]). However, as with any map the layout of buttons leaves significant white space and does not communicate much information (in comparison to the physical space it occupies). The tradeoff seems worthwhile since the interface does not compete for space on a computer desktop - only otherwise unoccupied wall space as a precursor or alternate to a monitor.

Even with little information communicated, privacy concerns must be taken into consideration as presence information may be considered too personal to share with the many lab visitors. The caricature on the button provides a visual cue without revealing as much as a photo would - a caricature is only easily associated with a person if the person is somewhat known to the user. While early versions of $\mathrm{OE}$ used caricatures created by a local artist, to enable rapid update the later versions used a series of easilygenerated graphical manipulations to create a similar lightweight image style.

The combination of the buttons with the lights allows a user to match a lab member with his online availability with a quick 
glance. However, only a limited amount of information can be shown with a light and button, and while lab regulars would know that the light shows online status, a visitor might assume that it shows presence or other information. Again, we must also be cognizant that having the information on lab users, even in a semi-public space not accessible to everyone, could present a security risk under certain circumstances.

During prototyping, we explored blinking the LEDs to convey more information, but when lab members saw the blinking, they protested that it was far too interruptive. The blinking was dropped, and the design was changed to include bi-color lights so that distinction can be made between offline, away, and online with minimal annoyance.

\subsection{Implementation}

Implementing the design introduced issues of information access and physical model building, which often impacted the nature of the final design. We needed to access the information from IM, control the phidgets, tie those two together with the proper logic, and build the model.

We accessed the MSN Messenger Client through its programming objects, which are monitored by our program for user status changes. The program stores information about the MSN Messenger status of the lab users, along with clock information, in order to be able to give the time that a user's status changed.

Phidgets, the physical widgets developed at the University of Calgary, are tangible interface elements used in creating realworld interfaces [3]. The four phidget boards (two for the buttons, one for the LCD, and one for the LEDs) are easily driven by the program with their own software package, which manages LED and LCD changes and collection of button pushes.

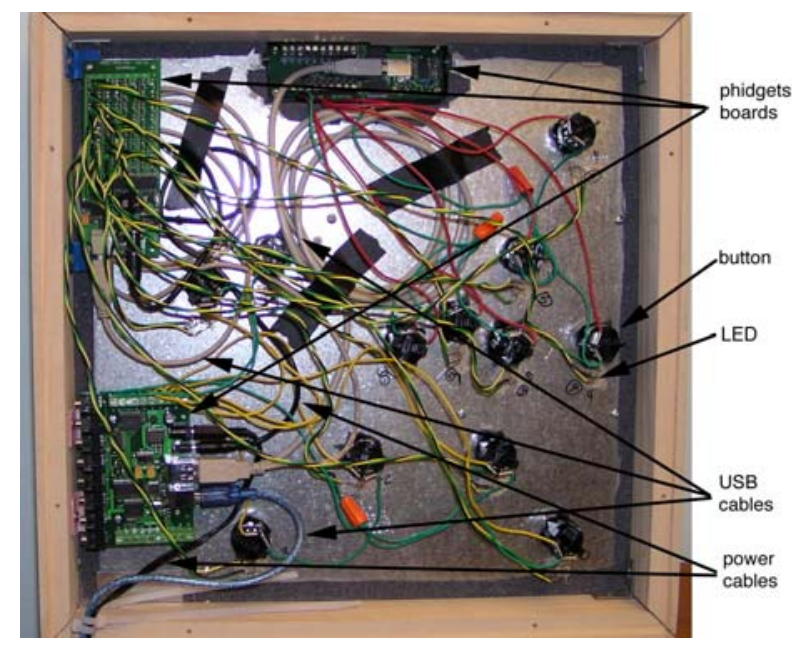

Fig. 2. Back of OE with back panel removed

\section{INITIAL USAGE REPORT}

Initial usage reactions were collected via two means: through an unveiling at a presentation open to all lab users (both regular ones with desks in the lab as well as occasional ones who only drop by the lab for meetings), and over a four-month period through observations and interviews of lab members and visitors.

\subsection{Presentation Feedback}

As the introduction of new technologies into the workplace can be disruptive, we were concerned that our user population would view $\mathrm{OE}$ as interruptive to their familiar work environment. However, there were no complaints about the potential for interruption from the final version of the display - a worry during design phases when we considered including the blinking and tickering of information. It is our hope that the participatory design session, frequent prototype response requests, and other channels for input helped not only to create a better product, but also to ease OE's introduction into the lab.

Many reactions involved functionality changes - the addition of new and different information, the augmentation of $\mathrm{OE}$ with "intelligent" processes to anticipate schedules, and so on. Generally, these suggestions had been considered and dismissed during design in an effort to simplify the design and purpose of the interface.

We worried that privacy would be a major concern, but despite our direct questions on the topic no complaints emerged regarding the type of information, and nobody requested that their information be removed from $\mathrm{OE}$-reflecting that for this situation, privacy is not a major issue for semi-public areas. Perhaps this is not completely surprising, as a semi-public display like $\mathrm{OE}$ is meant to strengthen connections within the community.

\subsection{Observations from the Field}

As of the time of this paper, the OE system has been in use in our lab for about four months. While numerous usage stories emerged from our observations and interviews, listed here are two representative stories about its use, highlighting some of the advantages $\mathrm{OE}$ provides as well as some downsides that must be tolerated or mitigated with other technologies.

Several graduate students in the lab were preparing a demonstration of a project that they were working on. Soon before giving the demo, they discovered they needed help configuring the server from another lab member who was neither present in the lab nor online elsewhere according to $O E$. While setting up their demo, the other lab member's OE light came on. They hurriedly went to a computer to message her, and together they were able to fix the problems by the time of the demonstration.

This illustrates how the constant information provided by OE can act to help communication between lab users. If the students had been relying on a deliberate checking of whether the other member was online, they would not have noticed that the information had changed. OE helps transform the lab space into a place where community can thrive, and it extends the boundaries of the place beyond the walls of the lab to include members who are currently working remotely. We also see how $\mathrm{OE}$ acts as a bridge to other technologies - encouraging use of IM once the appropriate time and place can be identified rather than trying to include all functionality within $\mathrm{OE}$.

One student came into the lab looking for any one of several graduate students to chat about an issue regarding the direction of his project. He noticed that all were away, but, upon pressing the buttons for each, saw that all had been away for about 5 minutes. As it was lunchtime, he concluded that they all had probably gone out together to lunch and decided to call one of their mobile phones. Upon catching up with the grad students, he 
was able to learn what he needed to know over lunch, and all returned to the lab afterwards, ready to be productive.

As lab members often move in groups-whether to class, lunch, or other events-OE can reflect that behavior and allow assumptions to be made by others. As students with common interests are generally co-located within the lab, their status lights and buttons are also close together within the map, making it easy to notice common light patterns and press the buttons in sequence. The small amount of information provided by OE upon a button press does not overly intrude on lab members' privacy by describing in detail their status, but it does supply clues as to their recent status to a point that an informed decision on how to acquire more information can be made. Again we see OE guiding users toward appropriate behavior with other technology-in this case, making a phone call to catch up with the person.

\subsection{Reactions Summary}

Regular lab users seemed to either be somewhat pleased with itlooking at it regularly, noticing who was in when they came through the hall or walked through the lobby area-but others rarely notice it. All users still rely on their IM buddy list on their screen to varying degrees - not surprising given the far greater power of IM systems. While we expect that comprehension of activities of lab members was increased based on anecdotal evidence, nobody dared speculate on whether comprehension was indeed enhanced.

Usage of IM differs greatly from person to person. Some used IM only in the lab, even switching their status to "away" when in the lab to indicate that they did not wish to be disturbed. Some are almost always connected somewhere, not logging off from one site until they log on to another. Others had to be all but required to use it (awkward for a academic research lab) and stopped after a brief usage period. However, regular users seemed to quickly realize whose display reliably reflected presence and whose did not. Unlike many CSCW systems, full buy-in from participants is not needed - even a couple of reliable indications of presence made the system useful to knowledgeable lab members.

As with many ubiquitous displays, we felt compelled to hang a sign on $\mathrm{OE}$ when it was first released, explaining its purpose and functions. We viewed this as something of a failure; ideally, such a system should be self-explanatory. However, as the semi-public display became more a part of the daily lives of the lab regularspart of an active place rather than a static space-the sign no longer seemed necessary. Even lab visitors seemed never to take the time to read it, instead choosing to poke and play with the interface to figure it out. Eventually, we removed the sign from the interface, allowing the interface - and the community-to speak for themselves.

An occasional visitor to the lab had the following observations. It was unclear what the lights and buttons represented, and since the LCD was not noticed at first, the button function could not be ascertained. Breakdowns are not unexpected for a semi-public display [6], especially upon first sight. However, even a person not familiar with the interface recognized the map metaphor and saw the functionality of the buttons, even in an augmented state.

\section{CONCLUSIONS}

This paper described the development of Online Enlightenment, a semi-public display showing IM online status for a community of users. OE is mounted on the wall in a lab housing about ten people, and it used mainly by the regular lab inhabitants but also by visitors. Reactions to the interface, collected over a four-month usage period, shows how ubiquitous interfaces designed for a tight community of users can enhance and support their interactions.

Simple design elements, like the familiar map metaphor, LEDs and LCDs, and caricature-augmented buttons, proved to be highly effective. We welcome but are cautious about ideas for new functionality that can be added, as we wish to keep focused the purpose of $\mathrm{OE}$. More information-dense designs that we considered or prototyped tended to overwhelm rather than appropriately inform.

Underlying the design of the $\mathrm{OE}$ application was a broader purpose: to assess methods used in creating systems like OE in transforming a structural space that inhibits interaction to a socially vibrant place that enhances it. Our approach allowed us to highlight positives and negatives of features in the designimportant during design and evaluation. The lessons learned from the creation process contribute to an evolving methodology for this type of interface.

\section{References}

1. De Guzman, E. S., Yau, M., Gagliano, A., Park, A., \& Dey, A. Exploring the Design and Use of Peripheral Displays of Awareness Information. In Proceedings of CHI 2004 1247-50.

2. Ganoe, C., Somervell, J., Neale, D., Isenhour, P., Carroll, J. M., Rosson, M. B., McCrickard, D. S. Classroom BRIDGE: Using Collaborative Public and Desktop Timelines to Support Activity Awareness. In Proceedings of UIST 2003 21-30.

3. Greenberg, S., Fitchett, C. Phidgets: Easy Development of Physical Interfaces through Physical Widgets. In Proceedings of UIST 2001, 209-218.

4. Grudin, J. Partitioning Digital Worlds: Focal and Peripheral Awareness in Multiple Monitor Use. In Proceedings of $\mathrm{CHI}$ 2001, 458-465.

5. Heir, M., Hoon, H., Terrell, G., McCrickard, D. S. Online Enlightenment: A Phidget Notification System for Online Status. Virginia Tech Department of Computer Science Technical Report TR-04-30, 2004.

6. Huang, E. M., Mynatt, E. D. Semi-public displays for small, co-located groups. In Proceedings of CHI 2003, 49-56.

7. Huang, E. M., Russell, D. M., and Sue, A. E. IM Here: Public Instant Messaging on Large, Shared Displays for Workgroup Interactions. In Proceedings of CHI 2004, 279-286.

8. Harrison, S., Dourish, P. Re-Place-ing Space: The Roles of Place and Space in Collaborative Systems. In Proceedings of CSCW 1996, 67-76.

9. Lederer, S., Heer, J.: All Together Now: Visualizing Local and Remote Actors of Localized Activity. In Proceedings of $\mathrm{CHI}$ 2004, 1107-1110.

10. McCarthy, J. F. and Meidel, E. S. ActiveMap: A Visualization Tool for Location Awareness to Support Informal Interactions. In Proceedings of HUC 1999, 158-170.

11. McPhail, S.: Buddy Bugs: A Physical User Interface for Windows Instant Messaging. In Proceedings of WCGC 2002.

12. Stasko, J., Miller, T., Pousman, Z., Plaue, C., Ullah, O.: Personalized Peripheral Information Awareness through Information Art. In Proceedings of UbiComp 2004, 18-35. 